\title{
Crystal growth and optical study of thalidomide
}

\author{
Mizuki Kira ${ }^{1}$, Koichi Tsutao ${ }^{1}$, Kun Zhang ${ }^{1}$, Yukana Terasawa1 ${ }^{1}$, Norio Shibata², Toru Asahi ${ }^{1,3}$ \\ ${ }^{1}$ School and Graduate School of Advanced Science and Engineering, Waseda University, 3-4-1 Okubo, \\ Shinjuku-ku, Tokyo 169-8555, Japan, \\ ${ }^{2}$ Department of Nanopharmaceutical Sciences and Department of Frontier Materials, Nagoya Institute of Technology, Gokiso, \\ Showa-ku, Nagoya 466-8555, Japan, \\ ${ }^{3}$ Research Organization for Nano and Life Innovation, Waseda University, 513 Wasedatsurumaki-cho, Shinjuku-ku, \\ Tokyo 162-0041, Japan \\ kira5-5@akane.waseda.jp
}

Thalidomide (TD) was first developed as a safe sedative hypnotic in 1958 and was prescribed for pregnant women to prevent morning sickness. However, TD was reported to cause the tragic side effect in 1960s: Babies born to mothers who took TD had small limb or malfunctioning organs. Nevertheless, TD has attracted much attention as an indispensable pharmaceutical compound because of its effectiveness in treating intractable diseases. Meanwhile, Blaschke et al. reported that only (S)-isomer of TD caused its teratogenicity [1]. This report has influenced pharmaceutical researchers, and then the importance of studies on chirality of drugs have been recognized. However, subsequent studies revealed facile racemization of TD in the aqueous solution [2,3], which led us to discuss the interpretation for Blaschke's report. Since then, many researchers in various scientific fields have been interested in the mechanism of teratogenicity of TD.

In 2010, the molecular target of TD was identified Cereblon (CRBN) [4], which forms an E3 ubiquitin ligase complex with damaged DNA binding protein 1 [5]. The structural and biochemical studies on the R- snd S-enantiomers of TD binding to CRBN has been reported, which has revealed that the S-enantiomer exhibits ca.10-fold stronger binding to CRBN compared to the R-enantiomer [6].

Furthermore, the important and significant study on TD have been reported [7, 8], which can demonstrate Blaschke's report. Meanwhile, experimental results on TD itself in the crystalline state were not sufficiently done. Especially, optical properties in TD crystals were not revealed because of the difficulty in growing the good single crystal. We have succeeded in growing thin single crystals of TD with sublimation methods and studying optical properties in them using the Generalized High Accuracy Universal Polarimeter abbreviated as G-HAUP, which enables us to measure simultaneously linear birefringence (LB), linear dichroism (LD), circular birefringence (CB; optical activity), and circular dichroism (CD) in anisotropic materials [9-11].

We will show wavelength dependencies of anisotropic optical properties, LB, LD, CB, and CD, in TD single crystals.

[1] Blaschke, G., Kraft, HP., Fickentscher, K., Kohler, F. (1979). Arzneimittel-Forschung, 29, 1640-1642.

[2] Nishimura K., Hashimoto, Y., Iwasaki, S. (1994). Chem. Pharm. Bull., 42, 1157-1159.

[3] Knoche, B., Blaschke, G. (1994). J. Chromatogr. A, 666, 235-240.

[4] Ito, T., Ando, H., Suzuki, T., Ogura, T., Hotta, K., Imamura, Y., Yamaguchi, Y., Handa, H. (2010). Science, 327, $1345-1350$.

[5] Fischer, E. S., et al. (2014). Nature, 512, 49-53.

[6] Mori, T., Ito, T., Liu, S., Ando, H., Sakamoto, S., Yamaguchi, Y., Tokunaga, E., Shibata, N., Handa, H., Hakoshima, T. (2018). Sci. Rep., 8, 1294.

[7] Maeno, M., Tokunaga, E., Yamamoto T., Suzuki, T., Ogino, Y., Ito, E., Shiro, M., Asahi, T., Shibata, N. (2014). Chem. Sci., 6(2), $1043-1048$.

[8] Tokunaga, E., Yamamoto, T., Ito, E., Shibata, N. (2018). Sci. Rep., 8, 17131.

[9] Kobayashi, J., Asahi, T., Sakurai, M., Takahashi, M., Okubo, K., Enomoto. Y. (1996). Phys. Rev. B, 53, 11784-11795.

[10] Tanaka, M., Nakamura, N., Koshima, H., Asahi, T. (2012). J. Phys. Appl. Phys., 45, 175303-175311.

[11] Nakagawa, K., Asahi, T. (2019). Sci. Rep., 9, 18453.

Keywords: Thalidomide; Crystal growth; Generalized High Accuracy Universal Polarimeter; Optical properties 